\title{
The challenge of electric mobility in digitized power grids
}

Heiko Fastje, EWE NETZ GmbH; I. Kolmsee, EWE AG

This manuscript was not available on completion of this publication.

Thank you for your understanding. 